\title{
El tutor y la tutoría en el proceso de formación de especialistas sanitarios en la comunidad de Madrid. Análisis e interpretación mediante grupos focales.
}

J esús Antonio Álvarez Sánchez ${ }^{1}$, María Dol ores Vicent García ², J osé Manuel Salamanca Escobedo, Fernando Pérez I glesias ${ }^{4}$, Miguel Carrasco Asenjo².

Hospital EI Escorial. Servicio Madrileño de Salud ${ }^{1}$. Agencia para la Formación, Investigación y Estudios Sanitarios. Consejería de Sanidad. Comunidad de Madrid ${ }^{2}$. Servicio de Atención Primaria. Servicio Madrileño de Salud. ${ }^{3} \mathrm{H}_{\text {ospital }}$ Severo Ochoa. Instituto Madrileño de Salud. ${ }^{4}$

Fundamento: El sist ema de formación de especialistas sanitarios (SFE) en nuestro país tiene una gran importancia en el mantenimiento de un Sistema de Salud de calidad. La evolución de dicho sistema ha ido configurando al tutor de los residentes en el eje sobre el que recae gran parte de la responsabilidad de la calidad del proceso formativo y de la competencia clínica de los especialistas titulados, sin que se hayan definido los requisitos que deberían acompañar dicha responsabilidad. El objetivo del estudio es explorar la opinión de los tutores respecto de sus responsabilidades y necesidades en el SFE, y en un contexto de tutoría planificada.

Métodos: La investigación se realizó mediante cuatro grupos focales formados por tutores de atención primaria y hospitales, donde los residentes realizan su formación.

Resultados: los discursos de los participantes se centraron en la definición del perfil asistencial y académico de los tutores, las características que debería reunir el proceso de selección y acreditación del tutor, $y$ en las responsabilidades, funciones y reconocimiento de la tutoría.

\section{Correspondencia:}

J esús Antonio Álvarez Sánchez.

Hospital EI Escorial. Servicio Madrileño de Salud.

Ctra. Guadarrama, Km. 17,600

28200 San Lorenzo de EI Escorial. Madrid, España

E-mail: alvagrau@yahoo.es
Conclusiones : se ha identificado claramente la falta de concordancia entre el valor que "en t eoría" se otorga a la docencia y la "práctica" de la misma, caracterizada por su subordinación a la asistencia, la escasez de recursos y la falta de reconocimiento de la labor docente. Se apunta la democratización y objetivación del proceso de designación del tutor, una cierta independencia de la tutoría respecto de la estructura asistencial, la potenciación de las Comisiones Docent es y Asesoras, el reconocimiento en baremos profesionales y el tiempo destinado a la tutoría como la mejor compensación.

Palabras clave: Educación médica; Programa de formación; Residencia; Tutor; Postgrado; Educación/ métodos; Sanidad.

Medical Specialist Training Prog ramme: views of trainers in the Community of Madrid. Analysis and interpretation by focal Groups.

Introduction: Medical specialist training programme (STP) has a great importance in the maintenance of high quality health care delivery in our Country. Programme's development has converted trainers into the main responsible for the quality of the training programme itself, and the clinical competence of the trainee. Never theless, the requirements for such a responsibility have not been yet defined. Evaluation 
of trainers' views about STP is linked with their responsibilities, and is placed within the context of the need for a more structural or planned approach to training.

Method: This research was conducted in the Community of Madrid, by means of four Focal-group. Trainers were selected from primary care and hospitals where STP occurs.

Results : Participants discussed about the academic and medical profile of the tutor, the characteristics of the selection and accreditation process of trainers, and about responsibilities, tasks and recognition of the tutorial.

Conclusions: A discordant between the theoretical and practical values assigned to the teaching staff has been clearly identified. The later was determined by its dependence respect to the clinical practice, the paucity of resources, and the lack of recognition of their role in the teaching establishment. Clearance-cut with objective nomination process, some independence of training in the clinical setting, emphasize the role of the Teaching Commissions, arrange timing to the training program, and the recognition in the professional career were pointed as possible solutions.

Key words: Education, medical; Training programmes; *Internship and Residency; Tutor; Postgraduate training; Teaching/*methods; Health care.

\section{INTRODUCCIÓN}

Existecierto consenso en el ámbito sanitario de que el sistema de formación de especialistas en nuestro país ha sido e el emento dave de la mejora de la calidad de la asistencia sanitaria que se presta a los ciudadanos. Dicho sistema se basa en un proceso de prác tica profesional programada y supervisada, a fin de al canzar, deforma progresi va, los conocimientos, habi lidades y responsabilidades necesarios para ejercer la especial idad de forma efi ciente. Aunque el sistema de formación está estructurado en distintos niveles en cuanto a la organización, planificación y supervisión de los períodos formativos (Ministerial, Comisiones Nacionales de Especialidad, Comisiones locales de Docencia), la responsabilidad práctica de su desar roIlo, seguimiento y evaluación recae sobre los tutores. El desarrollo nor mativo del modelo de Formación de Especialistas ${ }^{1-2}$ atribuye a la tutoría, una responsabilidad notabley un auténtico compromiso en la preparación de los futuros profesionales de la Sanidad. Sin embargo, no es infrecuentequeen esteámbito coexis- ta un sentimiento vocacional en la práctica de la docencia que, si bien es importante y ha sido una de las características del sistema, no deja deser entendido por algunos como la asunción de ciertas funciones, que no par ecen complicar esencialmente el desempe ño profesional diario. Lejos deesta concepción, la responsabilidad que implica asumir el cargo de tutor, le conviertefuncionalmente en garante dela cal idad del proceso formativo que se imparte en la Unidad Docente. También es responsable respecto de los futuros profesionales sanitarios, por cuanto el desarrollo de sus funciones implica, cuanto menos, un conodmiento con detalle del Programa de la especialidad, capacidad para adecuar los recursos de la Unidad al desarrdlo de ese programa o la búsqueda de alternativas para complearlas, y competencia para realizar una evaluación y seguimiento de la calidad de la docencia impartida en la Unidad Docente

Hasta el momento, la docencia se ha entendido como algo intrínseco al trabajo diario de la Unidad Asistencial, en la que participan con mayor o menor intensidad todos los miembros de la misma. El profe sional en formación, por un lado, está bajo la supervisión de un tutor y, por otro, se incorpora a la actividad laboral en un Servicio asistencial, con su organización, recursos y objetivos, bajo la responsabilidad del J efe de la Unidad. Esta dualidad, tan característica del modelo de for mación M.I.R., también define gran parte de los problemas surgidos en la selección de tutores, y los conflictos de competencias que frecuentemente se plantean con la J efatura del Servicio. EI proceso de designación del tutor será más o menos importante para la Unidad Docente, y estricto en cuanto a los criterios de acreditación eindependencia, según y cómo la balanza seindine hacia la vocacionalidad sin exigencias o la profesionalidad estricta.

La experiencia acumulada en los años que lleva funcionando la formación de especialistas por el sistema de residencia, no par ece dejar dudas respecto a la necesidad de establecer un conjunto de criterios para la acreditación de tutores, y dotarlo de los instrumentos necesarios para asegurar el desarrollo de los programas docentes y vel ar por la calidad del proceso de formación ${ }^{3-6}$. El peso que confiera el proceso de selección de tutores a los elementos cur riculares dásicos, al perfil dínico, investigador o docente, o su indusión dentro de la car rera profesional condicionará la atracción que la tutoría tendrá sobre los profe sionales. Del mismo modo, no hay que olvidar quelos tutores, aún siendo expertos en su respectiva especialidad médica, en general no han dispuesto de un entr enamiento específico para formar a otros compa- 
ñeros, desconociendo los diversos métodos docentes utilizables en la formación deadul tos, el aprendizaje basado en la resolución de problemas, la investigación en acción, los instrumentos de evaluación de la Certificación o la competencia, o la evaluadión etnográfica ${ }^{7}$ del proceso de for mación, sólo por citar alguno de ellos. Este tipo de formación ya ha comenzado a desarrollarse en al gunas Comunidades Autónomas como Cataluña, Madrid y Andalucía ${ }^{8}$. A estos factores hay que añadir uno más: no todos los "actores" que intervienen en la docencia entienden de la misma forma la figura del tutor. Depende de condicionantes locales, profesionales y personales, no siempre bien conocidos, pero en todo caso variableen los diferentes servicios asi stenciales y en distintos Centros, y que condiciona en gran medida sus funciones, capacidad para desarrollarlas e independencia en su trabajo.

Todas estas reflexiones enmarcan un contexto de trabajo, en el que las dificultades para dirigir y supervisar la formación del residente, la forma diferente de entender la figura del tutor por parte de los actores que intervienen en ese proceso formativo, las reflexiones sobre la calidad del sistema de formación de Especialistas Sanitarios, y las limitaciones y problemas del sistema actual de evaluación de los residentes, definen en gran medida el papel que debe jugar el tutor en todo el proceso formativo, como el eje funcional sobre el que gravita todo el sistema de evaluación del residente en formación, y con ello, de todo el sistema MIR, y un punto central y habitual de discusión en Comisiones Docentes y Asesoras en las que participan los investigadores.

Así pues, el objetivo del estudio es conocer y comprender la opinión de los tutores y otros responsables de la formación de especialistas sobre el perfil del tutor y sobre las condiciones necesarias para desarrollar la tutoría, identificando aquellos componentes personales y profesionales del tutor que, a su juicio, dan el sentido actual a la tutoría en la formación de especialistas sanitarios dentro del organigrama asistencial. La finalidad del estudio es promover intervenciones institucionales que permitan mejorar la formación de los futuros profesionales y con ello, la asistencia que se presta a la población.

\section{SUJETOS Y MÉTODO}

Para la consecución de los objetivos del estudio se ha elegido grupos focales. El estudio trata de responder a los interrogantes e inquietudes expresadas por los tutores de la Comunidad de Madrid, que han sido recogidas por los investigadores, mediante su participación en la Red de Comisiones Docentes y Asesoras de Formación de Especialistas Sanitarios de la Comunidad de Madrid y en los debates realizados en el Foro de Presidentes y Representantes Autonómicos de las comisiones citadas, órganos de participación de los distintos actores del sistema de formación de residentes.

\section{DISEÑO DEL ESTUDIO}

- Técnicas: se optó por la utilización de entrevistas grupales (grupo focal) y observación participante de los investigadores, con el propósito de aumentar el rango de información recogida y su calidad ${ }^{9-15}$. El campo de estudio selimita a la Comunidad de Madrid.

- Seleción de los participantes: Los participantes fuer on seleccionados de las Unidades Docentes de los Centros acreditados para la formación de especialistas sanitarios. Se consideró elegible a los tutores y J efes de Estudios hospitalarios y tutores y Coordinadores de Unidades Docentes de Atención primaria. Para la selección de los participantes en el estudio se estableció una ponderación, atendiendo al tamaño de Centro docente en cuanto a número de especialidades acreditadas para la docencia postgraduada y al número total de profesionales en formación adscritos a cada Centro Docente, para favorecer que gran parte de los centros acr editados estuvieran representados en los grupos. J unto con los criterios de ponderación anteriores se estableció un máximo de participantes por Unidad Docente y Área Sanitaria. EI número final de participantes fue de 33 , quesedistribuyeron en cuatro grupos de discusión. El tiempo que llevaban realizado tareas de tutoría en la formación de especialistas fue muy variable, siendo el mínimo de apenas unos meses, aunquetodos procedían de este sistema de formaci ón y por lo tanto conocían plenamente su funcionamiento. Todas las personas incluidas en los grupos disponían de experiencia y capacidad para tratar los objetivos planteados.

- Convocatoria de los participantes: Como paso previo al muestreo, se realizó una difusión del proyecto, utilizando un canal preestablecido, a través de las reuniones habituales del Foro de Comisiones Docentes y Asesoras, y directamente a cada Unidad Docente. La Comunidad de Madrid está organizada en 11 Áreas Sanitarias, cada una de las cuales cuenta con una Comisión Asesora de Medicina Familiar y Comunitaria, y uno o más Centros Hospitalarios acreditados para la formación de especialistas que tienen una Comisión de Docencia. Actualmente, el número de Comisiones de Docencia es de 20. Se cur- 
Tabla I. Características de los grupos.

\begin{tabular}{cccccc}
\hline \multirow{2}{*}{ Grupo } & $\begin{array}{c}\text { Tutor hospital } \\
\text { Tutor MFC }\end{array}$ & Área Sanitaria & \multicolumn{2}{c}{$\begin{array}{c}\text { Jaños servicio } \\
\text { Adjunto }\end{array}$} & \multicolumn{2}{c}{ Total } \\
\hline 1 & $7 / 2$ & $1-2-4-5-10$ & $3 / 9$ & $2 / 7$ & 9 \\
2 & $6 / 2$ & $1-2-5-6-10$ & $2 / 6$ & $2 / 6$ & 8 \\
3 & $6 / 2$ & $1-3-4-7-11$ & $1 / 7$ & $2 / 6$ & 8 \\
4 & $6 / 2$ & $1-4-7-9$ & $3 / 5$ & $2 / 6$ & 8 \\
\hline \hline
\end{tabular}

Tabla II.

\begin{tabular}{|c|c|}
\hline Preguntas exploratorias generales & Conceptos preliminares y escenario de discusión \\
\hline $\begin{array}{l}\text { ¿En qué consiste ser tutor M.I.R. } \\
\text { y cual es el valor asignado en } \\
\text { el mundo asistencial y docente? }\end{array}$ & $\begin{array}{l}\text { • ¿Quién puede ser elegido tutor? } \\
\text { ¿Cual es la importancia de realizar una labor asistencial activa? } \\
\text { ¿Qué importancia tiene la antigüedad, o la vinculación contractual? }\end{array}$ \\
\hline $\begin{array}{l}\text { ¿Cuál debe ser el perfil asistencial } \\
\text { y académico del tutor y de la tutoría? }\end{array}$ & $\begin{array}{l}\text { •¿Qué méritos debe reunir el tutor? } \\
\text { ¿Es necesario establecer unos méritos mínimos? } \\
\text { ¿De qué categoría deben ser los méritos (académicos, profesionales...)? } \\
\text { ¿Cómo se integra la Investigación, la formación específica en Metodología o en técnicas docentes? } \\
\text { ¿Cómo ponderar los méritos cuantitativos y cualitativos? } \\
\quad \text { ¿Cómo se proponen y seleccionan los tutores? } \\
\text { ¿Qué papel debe tener el Jefe del Servicio en la designación de tutores? } \\
\text { ¿Cual debe ser la participación del resto de los profesionales de la Unidad asistencial? } \\
\text { ¿Cual es el papel asignado a la Comisión de Docencia y a los residentes? } \\
\text { ¿Cómo detectar los elementos que condicionan la propuesta del tutor? } \\
\text { ¿Cual es el grado de responsabilidad del Jefe de Servicio, Comisión de Docencia, Residentes, Miembros del Servicio } \\
\text { y Dirección en la selección? } \\
\text { ¿Cómo participan todos estos actores en el proceso de selección? } \\
\text { ¿Cómo dirimir los conflictos en el proceso de selección de tutores? } \\
\quad \text { ¿Cómo se evalúa la calidad de la tutoría? } \\
\text { ¿Cómo se establecen los medios y la estructura mínimos exigibles? } \\
\text { ¿Cómo se establecen unos objetivos explícitos para la tutoría? } \\
\text { ¿Quién debiera controlar el rendimiento del tutor y con qué periodicidad y proceso? } \\
\text { ¿Cual es la participación de los Residentes y la Unidad Docente en su conjunto en la evaluación del tutor?. } \\
\text { ¿Qué mecanismos de control deben existir en la formación asistencial y docente del residente? } \\
\text { ¿Cual debe ser el mecanismo de sustitución de tutores? }\end{array}$ \\
\hline $\begin{array}{l}\text { ¿Qué elementos pueden definir } \\
\text { la posición del tutor en el organigrama } \\
\text { asistencial y docente, y condicionar } \\
\text { el desempeño de sus funciones? }\end{array}$ & $\begin{array}{l}\text { • ¿Cómo influye la situación jerárquica del tutor en la calidad de la tutoría? } \\
\text { ¿Como afecta la ausencia de un reconocimiento explícito al tutor dentro del organigrama hospitalario al ejercicio } \\
\text { de las funciones? } \\
\text { ¿Cómo diferenciar las necesidades de la tutoría del reconocimiento de los tutores? } \\
\text { ¿Cual debe ser la forma de compensación al tutor? } \\
\text { ¿Qué responsabilidad tienen la Comisión de Docencia y la Dirección en las decisiones del tutor en materia formativa? } \\
\text { ¿Cómo se dirimen los conflictos? }\end{array}$ \\
\hline
\end{tabular}

saron 38 invitaciones para partidipar a las Comisiones de Docencia.

- Lugar de reunión: por razones de oportunidad, disponibilidad de espacios y eficiencia se decidió realizar los grupos simultáneamente, en distintas salas ubicadas en un centro docente, fuera de su lugar habitual de trabajo a fin de conseguir un marco más neutral.

\section{DESARROLLO DE LOS GRUPOS}

- Se trabajó con auatro grupos focales. El número de participantes por grupo osciló entre 7 y 9. Las carac- terísticas de los grupos se muestran en la tabla 1. Se eligieron grupos focales equilibrados en cada una de las variables. Estos grupos focales permiten explorar las opiniones y actitudes de los profesionales antelos objetivos del estudio, mediante la interacción entre los integrantes ${ }^{9}$. Para facilitar el trabajo en los grupos, se facilitó el guión semi-estructurado dela tabla 2. En cada grupo se incorporó un moderador con la intención de realizar una observación participantee iniciar el grupo medianteuna facilitación verbal.

- Después de la presentación por parte del mode rador, en cuanto al objetivo general del estudio, defi- 
niendo los conceptos preliminares del tema y planteando el escenario de discusión, el grupo se inició mediante una pregunta exploratoria general en cada uno de los tres objetivos generales del guión. El moderador del grupo debe facilitar la exposición de los participantes en la discusión, que hablen con total libertad y expongan su experiencia y vivencias, que fluya lo acaecido en la comunidad docente. Al finalizar el periodo de discusión, cada participante debía resumir los aspectos considerados de mayor utilidad e importancia. El discurrir de la exposición de los difer entes ponentes debía mostrar el panorama en $\mathrm{A}$ cual los tutor es desarrollan su tr abajo docente dentro del mundo asistencial, desde la perspectiva del tutor hospitalario y del tutor deAtención Primaria.

- Los investigadores que participaron en los grupos de discusión como moderadores, seconsiderar on como actores en el contexto en el que se desar rolla la tutoría, puesto que todos eran miembros de Comisiones Docentes y Asesoras con distintas responsabi lidades en la formación especializada, sin embargo, el perfil profesional de cada moderador fue diferente para cada grupo (dínico médico, quirúrgico, asistencia primaria, epidemiología, administración central), para evitar sesgos influidos por una excesiva implicación pr ofesional del investigador en el tema. Un papel fundamental de los observadores era facilitar la exposición de los participantes en la discusión, que hablaran con total liber tad y expusier an su experiencia y vivencias respecto del tema de discusión planteado. Para realizar dicha labor los moderadores disponían de un cuestionario semi-estructurado, el aborado a partir de sus observaciones previas, que se utilizó como guión de la entrevista de los grupos focales.

- Se registraron las observaciones e interpretación de los participantes de los grupos. El moderador de cada grupo anotaba tanto las respuestas como el componente emotivo que se ligaba a cada tema. Se añadieron las impresiones y experiencias de cada uno de los investigadores, aunque diferenciándolas de las observaciones de los participantes. Una vez alcanzada una esquematización que resumía las observaciones realizadas en el grupo y cómo percibían los interrogantes planteados, se realizó un nuevo cido de discusión incluyendo a todos los participantes como un único y nuevo grupo, para analizar y contrastar las observaciones de cada grupo, y captar nuevas aportaciones relevantes para el análisis posterior. En este grupo se induyó un quinto investigador. Siguiendo un criterio de saturación, el proceso de discusión se mantuvo hasta que se consideró que las nuevas observaciones no aportaban información adicional. El tiempo total asignado a la discusión del escenario fue de doce horas, ya que los grupos se constituyeron como tales en dos ocasiones, en días consecutivos.

\section{ANÁLISIS E INTERPRETACIÓN DE LOS DATOS}

- Consecuentemente con la metodología cualitativa el análisis se realizó en distintas fases: en primer lugar, análisis previos de las observaciones de investigadores en el contexto natural, posteriormente, los análisis preliminares de los textos obtenidos en los grupos de discusión por cada uno de los investigadores y, por último, análisis final se realizó mediante consenso de las interpretaciones de los investigadores. EI análisis se centró en los siguientes puntos: 1) conocer cual es el concepto y el valor asignado a la figura del tutor por los encargados de la formación de especialistas sanitarios; 2) establecer el perfil asistencial y académico que debe tener el Tutor y la Tutoría docente postgraduada; y 3) explorar los elementos que definen las relaciones del tutor en el organigrama asistencial y la Unidad Docente.

\section{RESULTADOS}

Los resultados muestran una síntesis de las opiniones de todos los grupos y los moderadores, a modo de concepción básica sobre aspectos comunes de la organización y el desempeño de la tutoría en las Unidades Docentes hospitalarias y de atención primaria. En la redacción se ha respetado la estructura de los bloques de discusión. Las citas textuales de los participantes aparecen en cursiva.

\section{- ¿El concepto y el valor asignado a la figura del tutor por los encargados de la docencia refleja lo que se le atribuye en la normativa?}

Destaca la forma homogénea de percibir la figura del tutor y el ejercicio de la tutoría docente por parte de los encargados de la formación. Todos acordaron, sin demasiada discusión, que la tutoría no está ade cuadamente valorada. Este sentimiento de falta de reconocimiento, se expresó en manifestaciones de "como un hermano menor en una gran familia con problemas". Aunque se reconoce la importancia de la Orden Ministerial, en cuanto recoge las funciones y responsabilidades del tutor, los participantes consideraron que era necesario contar con un soporte más explícito respecto a la capacidad de decisión y dependencia jerárquica del tutor, y sobre todo adaptado a la presión asistencial de los centros acreditados para la docencia. Esta enumeración de funcio- 
nes, entrelazadas con las asistenciales, resulta des proporcionada para el apoyo que finalmente reciben para desarrollarlas.

\section{- ¿Cuál es el perfil asistencial y académico que debe tener el Tutor y la tutoría? ¿Quién puede ser elegido tutor?}

La docencia postgraduada es percibida como algo intrínseco de los centros asistenciales, integrada en el trabajo diario, y en la que participan la mayoría de los miembros de la misma con mayor o menor intensidad. Por tanto debe existir una responsabili dad general para toda la Unidad Docente expresada como un compromiso con la formación, y otra per sonalizada, en la cual la responsabilidad máxima y final corresponde al tutor. En consecuencia, y como norma general cualquier profesional integrado en la labor asistencial normal, podría ser el egido tutor de esa Unidad. No obstante, se considera deseable que e tutor cumpla otros requisitos mínimos, entre ellos, el tiempo que el tutor lleva trabajando en la misma, pero más que de antigüedad se propone considerar la experiencia o "tiempo de permanencia en el Centro", por cuanto ello implica un conocimiento del medio en el que se trabaja, con sus peculiaridades asistenciales y personales, y el establecimiento de relaciones humanas y profesionales que faciliten el paso del residente por los diversos Servicios. EI tiempo mínimo sería definido por la propia Unidad Docente.

Tampoco una vinculación temporal debe exduir al profesional para asumir la tutoría, siempre que se cumplan las exigencias de "tiempo de permanencia en el centro" y que una vez asumida la tutoría este garantizada la continuidad el tiempo necesario para completar el cicl o formativo de la especialidad de que se trate. En cualquier caso, es una relación que el facultativo en formación debería conocer.

En opinión de los participantes, no es recomenda ble que los J efes de Servicio o Coordinadores de Equipo de Atención Primaria sean los tutores. Esta el ección sólo debiera producirse ante circunstancias excepcionales, si bien, ello no debe impedir que el J efe de Servicio participe en la formación de los residentes como un miembro destacado de la unidad docente. Las razones que fundamentan esta opinión ven en ella un factor de conflicto en el desempeño de sus funciones, y sobre todo en la posible falta de tiempo de dedicación que, habitualmente, pueden tener estos profesionales con los residentes. Las numerosas atribuciones y compromisos de la jefatura, entre las que se encuentra la gestión eficiente del propio servicio para garantizar la respuesta a la demanda de servicios, limitan la disponibilidad para una supervisión directa del residente, e interfiere con la formación en si misma, por cuanto lógicamente siempre predominaría la asistencia sobre la formación. También resulta difícil comprender cómo se controlaría la tutoría ejercida desde la propia jefatura, en cuanto al establecimiento y seguimiento de los objetivos docentes, que dada la organización jerárquica actual, sólo podría proceder de la Dirección del Centro.

En el caso hospitalario, en cualquier caso, es reconocido que la calidad de la formación impartida en una Unidad Docente dependerá en gran medida en la mutua confianza y responsabilidad del J efe de la Unidad y el tutor, junto con los residentes. La Comisión de Docencia debe contar con la suficiente capacidad operativa y decisión para jugar un papel más activo en este proceso. Como complemento, se considera importante que el tutor dependa funcio nalmente dela Comisión de Docencia. Una solución a esta aparente discrepancia de intereses que facilitara la necesaria independencia del tutor, sería la elaboración de un programa pactado entreel J efe de Servicio, el tutor y la Comisión deDocencia, antes de la incorporación del residente, que implicara un compromiso, una guía de actuación, y una delegación de responsabilidad en el tutor.

En el caso de los tutores de Medicina Familiar y Comunitaria (MFC) parece que la solución propuesta en el apartado anterior ya se está aplicando en algunas áreas con bastante éxito. Los tutores dependen funcionalmente de la Comisión Asesora y disponen de un programa o protocolo docente pacta do entre los tutores y el coordinador de equipo en cada centro de salud y con el coordinador de la Unidad Docente de MFC.

En cualquier caso, siempre subyace el problema dela per manentesubor dinación a la asistencia, cada vez con un mayor peso, que cerca la capacidad operativa de los tutores y de la Comi sión de Docencia para promover cambios que desarrollen y mejoren la docencia. Por ello, es necesario buscar una cierta independencia en el trabajo de tutor como docente respecto a las necesidades del trabajo diario.

\section{- ¿Qué méritos deben contar en la selección de tutores?}

La mayoría de los participantes perciben que es necesario establecer un perfil adecuado, tanto en la vertiente profesional como docente $\mathrm{Al}$ mismo tiempo, los criterios de elegibilidad y de selección de tutores que se establezcan no deben llevar a puntos extremos que provoque la falta de especialistas dis- 
puestos a ser tutores. Se huye de criterios sól o curriculares cuantitativos determinados por baremo, los cuales podrían definir un perfil exdusivamente académico, ni tampoco por aspectos subjetivos como la cercanía con el J efe de Servicio. Más bien, consideran que lo que importa es el balance cualitativo y cuantitativo. No cabe duda que el tutor debe ser un profesional actualizado, pero ante todo se piensa que e tutor debereunir unas determinadas cualida des "humanas" relacionadas con su capacidad de comunicación, de relación con los residentes. Por coherencia, su participación en formación continua da y actualización profesional debería mantener una cierta continuidad, con asistencia a cursos espe cíficos de la especialidad, formación en habilidades docentes, comunicaciones a Congresos y publicacio nes científicas. Por el momento, para los tutores hospitalarios, se considera poco ventajoso optar por un baremo cuantitativo, a modo de concurso, de cada uno de los puntos mencionados para poder optar a la plaza de tutor (hay que tener en cuenta que se están produciendo situaciones muy dispares, en cuanto a la disponibilidad de tutores). En los hospitales cada vez es más difícil conseguir candidatos, en cambio en Atención Primaria se está produciendo una tendencia inversa, con más tutores de los necesarios. En cualquier caso, siempre podría optarse por unos mínimos para discriminar entre varios candidatos. Un aspecto muy val orado es la experien cia previa como tutor y el reconocimiento de haber ejercido una buena tutoría, sin olvidar la posibilidad de que sea el residente el que elija al tutor entre los que cumplan los criterios mínimos.

\section{- ¿Cómo debe ser el proceso de selección de los tutores?}

En MFC comienza con la solicitud del profesional que desea ser tutor, aunque en ocasiones es el Coordinador el que inicia un proceso para que se presenten candidatos, sobre todo si existe un déficit de tutores que ponga en peligro la continuidad de la docencia. El proceso debe ser dirigido por la Comisión Asesora (establecimiento de ariterios), contando con un informe de responsable de la Unidad que acredite que los candidatos reúnen los criterios. Los casos de conflicto deben dirimirlos la Comisión Asesora.

Sin embargo, en el caso hospitalario, se opina que e jefedel servicio debeiniciar el proceso, al ser quien primero debe reconocer la necesidad de nombrar un tutor en esa Unidad. La propuesta formal del jefe de servicio será resultado de un proceso participativo de todos los miembros de la unidad, incluidos los residentes, que debería remitirse con un informe del jefe de Servicio, referente a datos objetivos del tipo cartera de servicios, o implicación en sesiones, etc. a la comisión docente, quien efectuaría la selección sobre la base de unos criterios de acreditación prees tablecidos, y redactaría la propuesta final a la Dirección para su nombramiento.

Aunque se considera deseable que el tutor cuente con la aceptación del jefe de servicio, esta aprobación no debe vincular el nombramiento, de modo que en caso de conflicto sea la Comisión de Docencia quien decida. Una minoría defendía la potestad exclusiva del jefe de servicio de hacer la propuesta a la Dirección del Centro.

\section{- Mecanismos de evaluación de la función del tutor. El proceso de recertificación.}

De igual modo que se establece un proceso de acreditación inicial, es importante contar con un mecanismo de evaluación periódica del rendimi ento de tutor y de la estructura docente La memoria anual del tutor dondedeben constar los planes indi vidualizad os deformación y la memoria deactivida des del residente serían los elementos básicos deesta evaluación, a los que unir al final de cada periodo formativo una encuesta devaloración del tutor reali zada por l os resi dentes.

También manifestaron que en cada unidad deben establecerse jornadas ó sesiones de control periódico del cumplimiento del programa y de los objetivos docentes, controles en los que deben participar el conjunto de la plantilla médica y los residentes.

En todos los grupos se expresó que para que fun cione todo este sistema de control dela calidad dela estructura docente, Ia Comisión Docente debe tener un papel más activo, como árbitro en todo el proceso de selección, de control de la actividad y rendimien to de los tutores, y en la solución de conflictos docen tes. Lógicamente, también debe poder cesar al tutor ante el incumplimiento de las responsabilidades y objetivos docentes marcados, a instancia de cualquiera de los que participan en la docencia o la reciben. Hay que tener en cuenta, que de acuerdo con la normativa vigente, la duración de la acreditación del tutor viene marcado por los tiempos de cada ciclo formativo.

En lo que se refiere a potenciar la actividad de la Comisión de Docencia, hay consenso en funciones como, a) desarrollar una guía para que el tutor realice adecuadamente sus tareas (Manual del Tutor); b) facilitar la formación de los tutores (formación pedagógica previa, formación continuada); c) evaluar el desarrollo de las tareas por parte de los tutores en 
función del programa docente de la unidad; d) recabar las memori as docentes por los tutores y residentes de cada servicio; y, e) propi ciar que se le faciliten medios a los tutores para ejercer sus funciones.

\section{- Explorar los elementos que definen} la integración del tutor en el organigrama asistencial y docente. Responsabilidades, funciones y tareas del tutor.

Con respecto a este punto, predomina la opinión de que antes de aplicar el programa docente, el tutor debe realizar una valoración de la capacidad docente del Servicio asistencial al que pertenece. El objetivo de esta valoración es proporcionar un entorno asistencial preocupado por la docencia y la investigación, que asegure un adecuado nivel de calidad docente para formar profesionales.

Siguiendo la normativa, el tutor sería el respon sable en cada unidad docente de supervisar el cum plimi ento del programa y los objetivos docentes dela especialidad, adecuando formación y capacidad asistencial. El documento de referencia es el programa docente editado por la Comisión Nacional de la Especialidad, adaptándolo a la realidad asistencial del Centro Docente, buscando las mejoras necesarias para proponerlas a la Comisión de Docencia, y elaborando un plan individual de especialización.

Los tutores opinan que es esencial, que la capacidad de programación se realice con cierta independencia. Sin embargo, el funcionamiento natural de los centros sanitar ios no facilita estetrabajo. Es habitual que el tutor encuentre una excesiva utilización asistencial del residente, que con frecuencia interfiere con el desarrollo del programa docente, en un Servicio no siempre con hábito docente o con una estructura de servicio desequilibrada que no permite un cumplimiento completo. La mayoría opinan que estos problemas se presentan con frecuencia y su solución es complicada. Esta situación es un elemento de conflicto entre las necesidades docentes y las necesidades asistenciales, contradicción que no pueden resdver ni el tutor ni la Comisión de docencia desde su posición actual en el organigrama. En este punto, la elaboración de un documento escrito que reflejara las necesidades asistenciales y la capacidad formativa ofertada por esa Unidad, podrí a facilitar la resolución de las discrepancias. Este documento, e programa docente dela uni dad, podría ser daborado por el tutor, firmado por e J efe deServicio y supervi sado por la Comisión deDocencia.

\section{- Mecanismos de seguimiento de la forma- ción del residente.}

A pesar de la dificultad que conlleva la elabora- ción de un plan individual de especialización por cada residente, con calendarios de rotaciones y objetivos, la consecución de este objetivo reduciría los conflictos con la asistencia.

Para facilitar una evaluación adecuada quizás fuese preciso limitar el número de residentes por tutor, adecuando este número a las características del hospital, el Servicio y la especialidad. Aunque algunos tutores expresaron que tentativamente se podría situar en 10 el máximo por tutor, este dato depende sobre todo de la especialidad.

Existió un acuerdo unánime en que la elaboración de la memoria anual de actividades por cada residente puede ser un elemento fundamental para el seguimiento y evaluación de los residentes. En ella se puede recoger los distintos datos de la actividad desarrollada y el rendimiento anual. La memoria debe ser visada por el tutor y refrendada por la Comisión de Docencia.

En opinión de la mayoría de los tutores, hasta el momento el libro del residente se ha mostrado como un documento que no recoge las peculiaridades de cada especialidad, las cuales en muchas ocasiones son los elementos que definen la formación al canzada, al mismo tiempo que engorroso en su cumplimentación. Una memoria de actividades junto a la evaluación anual por parte del tutor ante la Comisión de Evaluación, se considera que sería una forma más efectiva de comprobar si se han alcanza do los objetivos planificados al comienzo de la for mación. Se insiste en que el tutor debe reunirse periódicamente con el residente y con los demás pro fesionales que participan en la docencia, en sesiones específicas de tutoría, y con el coordinador de la Unidad Docente y el jefe de estudios para evaluar $\mathrm{e}$ cumplimiento de los objetivos docentes.

\section{- Reconocimiento social del papel del tutor.}

Hay que encuadrar el reconocimiento del tutor en la situación actual de los Centros asistenciales en cuanto a su organigrama, misión, carga asistencial y tablas retributivas. Existe el sentimiento entre los tutores que son valorados por sus residentes, que hay un cierto reconocimiento de su trabajo por la Comisión de Docencia y por algunos J efes de Servicio, pero en ningún caso por la Administración Sanitaria ni por la Dirección de los Centros.

Al analizar el modo de reconocer la labor del tutor seplantea diferenciar entre, a) los medios materiales y humanos que deben formar parte de las Unidades docentes, los cuales son elementos propios de la estructura docente de cada Centro, con independencia de los medios dedicados a la estructura asisten- 
cial, y como tales deberían ser considerados entre los mínimos para la acreditación, ninguno de las cuales puede ser considerados en sí mismos como retributivos, sino más bien como soporte para poder desarrollar las labores docentes; y b) el reconoci miento en sí mismo, es decir la compensación en baremo de méritos, compensadiones económicas (becas, ayudas para asistencia a congresos, retribución adicional, ...), días de libranza, etc.

Es evidente que para realizar estas tareas hace falta tiempo, pero con pocas excepciones, la tutoría se ejerce sin reconocimiento de un tiempo de dedicaci ón específico para tal función. En opinión de los entrevistados, dada la continuidad de la asistencia, la forma más apropiada para realizar esta liberación sería mediantejornadas completas en lugar dehoras por día. Al mismo tiempo se reconoce que esta liberación parcial del tutor puedegenerar cierta conflictividad entre profesionales, al recaer la labor asistencial del tutor sobre otros compañeros, quienes por otra partetambién participan, aunque de otra forma en la formación. Las tareas de tutor deben desarrollarse durante su jornada or dinaria de trabajo y para esto deben habilitarse horarios espećficos bien definidos para cumpli rlas, evitando que su trabajo habitual se cargue a los demás profesionales.

Existe acuerdo general de que se deben buscar formas de compensación para incentivar e trabajo de los tutores, si bien hubo di scr epancia sobre cual sería la mejor fórmula en la situación actual. La valoración del trabajo del tutor a través de una compensación económi ca complementar ia puede introducir el ementos distorsionadores que influyan induso en el pape del tutor, que atraiga a personas interesadas en $\mathrm{d}$ aspecto económico y con un interés marginal en la docencia. En cualquier caso nunca debiera incluirse como un elemento de la productividad variable vinculado a objetivos asistenci al es. En estos casos la participación de los residentes en la evaluación de los tutores puede jugar un papel regulador fundamental.

En cuanto a la pregunta de sí disponen los tutores de medios apropiados para dispensar este tipo de formación, actualmente, y en una gran parte de las Unidades docentes, la respuesta en general no puede ser afirmativa, ya que existen situaciones muy dispares.

La dotación de medios técnicos adecuados, de recursos económicos para realizar cursos de forma ción complementaria de los resi dentes, de recur sos ó actividades para perfeccionar la capacidad docente del propio tutor, etc., es una forma indirecta pero no despreciable, dereconocer el papel delos quesededi - can a la formación. Esta forma de reconocimiento, a través de una dotación de medios, con un costo eco nómico concreto, así como la posible retribución de las tareas docentes realizadas en tiempo extra, debiera dar lugar en un futuro próximo, a la apertu ra en los Presupuestos sanitarios, de un apartado específico dedicado a la docencia, como forma de garantizar la cobertura de los objetivos docentes y al mismo tiempo de eval uar costos.

Finalmente, se expresó que es innegable que el tiempo de práctica docente exige cuanto menos una valoración específica de la duración y permanencia en las tutorías en los baremos para el acceso a dis tintas plazas profesi onales, como mérito asistencial, y como reconocimiento en la carrera profesional .

\section{DISCUSIÓN}

En los resultados de este trabajo, se aprecia una razonable satisfacción de los profesionales que participan en el Sistema de formación de especialistas sanitarios, en cuanto, consideran, es capaz de formar profesionales que desarrollan su trabajo con competencia, y por tanto cumple los objetivos para los cuales había sido diseñado. Pero al mismo tiempo, se percibe que el cometido de los responsables locales de planificar y supervisar el desarrollo del proceso formativo no está adecuadamente val orado, aparece desacoplado respecto de la organización asistencial, y por ello tampoco está suficientemente reconocido. Como consecuencia, este trabajo parece que se realiza gracias a que existe un gran voluntarismo y por la dinámica adquirida hasta el momento por el sistema. La asignación de funciones y responsabilidades realizada por la Orden Ministerial no se corresponde con la suficiente dotación de recursos y capacidad operativa. No obstante, si se reconoce que esta normativa permite un nuevo marco de relaciones entre los actores responsables de la formación, en especial en lo referente a la oferta docente de las Unidades Asistenciales, la Comisión de Docencia y Asesora, los tutores y los residentes, y los mecanismos de control y evaluación, sin demasiados cambios estructurales en el sistema. De esta forma, los participantes han identificado tres factores de inestabilidad en el sistema de formación en el ámbito local: el posicionamiento del tutor en el organigrama asistencial, la subordinación a la asistencia, y el papel de la Comisión de Docencia. A ello habría que añadir, la insuficiente dotación de recursos para la docencia en los Centros. 
Si bien se recon oce que la principal finali dad de los hospitales y centros de salud es la asistencia sanitaria, no es menos cierto que legalmente estos centros deben desarrollar todo su potencial para la formación de los profesionales de salud y para la investigación (Ley General de Sanidad). En el caso particular de la formación de especialistas, existe además un compromiso establecido en el momento que se acredita el centro y se ofertan plazas. Por tanto, la formación de los especialistas no debe estar "subor dinada a la asistencia" sino "integrada" en ella. En estos momentos predomi na la "reali dad asistend al", en cuanto a las cargas de trabajo y las fluctuaciones de la demanda, y es a estos factores a los que se supedita la capacidad final para ofrecer un entorno asistencial preocupado por la docencia y la investigación. Para aligerar esta presión los tutores y jefes de estudio buscan una cierta independen da en su trabajo como docentes respecto a las necesidades del trabajo diario. En el caso hospitalario, quizás pudiera conseguirse mediante la elaboración de un programa pactado entre el J efe de Servicio, el tutor y la Comi sión de Docencia, antes de la incorporación del residente, que i mpl icaría un compromiso de la Uni dad con la formación, una guía de actuación para lograr unos objetivos, y una delegación de responsabi lidad en el tutor. El equivalente en Medicina Familiar y Comunitaria, sería un programa o protocolo docente pactado entre los tutores y el coordinador de equipo en cada centro de salud y con el coordinador de I a Unidad Docente (equivalente al J efe de estudios). Siguiendo la normativa, el tutor es el responsable en cada unidad docente de supervi sar el cumplimiento del programa y los objetivos docentes de la especialidad, adecuando formación y capacidad asistencial. La elaboración de un documento escrito que reflejara esa adecuación docente, buscando el equilibrio entre Ias necesidades asistenciales y la capacidad formativa ofertada por esa Unidad, podría facilitar la resolución de las discrepancias que en algún momento aparecen. De esta forma se diferencia de manera explícita dos responsabilidades: una responsabilidad general para toda la Uni dad Docente, y otra per sonali zada, en la cual la responsabilidad máxima y final corresponde al tutor. Del mismo modo que el tutor debe evaluar periódicamente con el resi denteel cumplimiento de los objeti vos docentes, tambi én debe reuni rse con los demás profesionales que parti cipan en la docencia, con el jefe o coordinador de la U nidad Docente y con el jefe de estu di os, real izando sesiones especí- fi cas de tutoría en las que participen todos los profesionales de esa Unidad.

La falta de una posición clara del tutor como tal en el organigrama asistencial, ha sido el origen de muchos de los conflictos existentes en las Unidades docentes y un límite para solucionarlos. Un componente importante en estos conflictos, es la forma desigual de entender la figura del tutor por parte de los diferentes actores que intervienen en ese proceso formativo, a veces con visiones incluso contrapuestas. En general, la representación conceptual del tutor vendría definido en los siguientes puntos: a) cualquier profesional integrado en la labor asistencial normal podría ser elegido tutor de esa Unidad; b) el tutor debe reunir una determinadas cualidades "humanas" relacionadas con su capacidad de comunicación, y de relación con los residentes; c) por coherencia, su participación en formación continuada y actualización profesional debería mantener una cierta continuidad, con asistencia a cursos específicos de la especialidad, comunicaciones a Congresos y publicaciones científicas; d) un aspecto muy valorado es la experiencia previa como tutor y el reconocimiento de haber ejercido una buena tutoría.

Un punto esencial en el funcionamiento del sistema docente, es que la Comisión Docente debe tener un papel más activo, decisorio y operativo, como árbitro en todo el proceso de selección, de control de la actividad y rendimiento de los tutores, y en la solución de conflictos docentes. Para ello es indispensable contar con un mecanismo de evaluación periódica del rendimiento del tutor y de la estructura docente de cada Servicio o Unidad, y del Centro en su conjunto.

Es evidente que para cumplir estas tareas hace falta tiempo. Sin embargo, con pocas excepciones, la tutoría se ejerce sin que se le reconozca un tiempo de dedicación específico. En este punto, y al hablar de la compensación a un tutor cada vez más profesionalizado, a quién se le exige más en planificación y evaluación, se debe diferenciar claramente lo que se considera medios materiales y humanos necesarios y exigibles en las Unidades docentes acreditadas, que deben ser el soporte del funcionamiento de la tutoría, de aquellas formas posibles de compensar o retribuir ese trabajo, ya sea en baremo de méritos, económica en sus diversas modalidades (becas, ayudas para asistencia a congresos, retribución adicional), o combinada. Es importante destacar que la mayoría de los tutores valoraron con recelo la introducción de incentivos económicos, no tanto por des- 
confianza ante lo simbólico que piensan sería ésta, sino porque puede complicar las relaciones dentro de la unidad (atractivo exclusivo económico, disminuir la colaboración de otros miembros de la unidad). Por ello se decantan preferentemente hacia el reconocimiento de un tiempo específico para la docencia, daramente diferenciado de lo asistencial. En todo caso, es innegable que el tiempo de práctica docente exige cuanto menos una valoración específica de las tutorías en baremo profesional y como reconocimiento en la carrera profesional. La dotación de medios técnicos adecuados en la unidad docente, de recursos económicos para realizar cursos de formación complementaria de los residentes, de recursos ó actividades para perfeccionar la capacidad docente del propio tutor, entre otras, aunque puede ser una forma indirecta de reconocimiento, es más un conjunto de necesidades mínimas en la estructura docente que deben ser exigidas para la acreditación. Por ello, parece necesaria la apertura de un apartado específico dedicado a la docencia en los Presupuestos sanitarios, como forma de garantizar la cobertura de los objetivos docentes y al mismo tiempo de evaluar costos.

\section{CONCLUSIONES}

1. Los tutores consideran que la docencia MIR no está suficientemente valorada. Es necesario identificar la docencia como una oferta más de la cartera de servicios y un objetivo de la Unidad asistencial, dotándola de una estructura estable, reconocida y aceptada por todos sus miembros. Para ello, cada Unidad asistencial debería realizar un análisis de adecuación entre la necesidad y demanda asistencial y su capacidad docente, que sería la base para la oferta docente dela Unidad y el marco de relaciones para resolver futuras discrepancias en la acre ditación docente y en las necesidades asistenciales.

2. El perfil del tutor, en cuanto al balance cuantitativo y cualitativo de méritos, debe ser definido por la Unidad docente, pero aunque es importante para la selección, en tanto que la tutoría no pueda ser ejercida con una categoría adecuada y reconocida, no debe suponer un límite al acceso a la docencia. En el proceso de selección del tutor deben participar todos los miembros en la Unidad. La profesionalización de la tutoría exige dedicación docente, responsabilidad profesional, capacidad de gestión y, por ello, una mayor cualificación formativa.

3. El tutor necesita independencia para planificar y coordinar la actividad asistencial y docente del profesional en formación. La elaboración de un documento al principio de la acreditación docente, recogiendo objetivos y planificación, puede ayudar a gestionar mejor los recursos y limitar conflictos con la asistencia. La memoria anual del tutor, con la programación y planes individuales de formación, programas de colaboración docente, la memoria de las actividades desarrolladas por el residente como elemento de evaluación de su rendimiento, y una evaluación de la estructura docente, podría servir de base para comprobar si se han alcanzado los objetivos planificados, detectar deficiencias y desviaciones formativas, e implementar un programa de calidad de la docencia.

4. Para garantizar el desarrollo de las funciones y responsabilidades de la tutoría, y dotar al sistema de formación de un proceso de control y evaluación de la calidad, es necesario reconocer y proporcionar a la Comisión Docente la suficiente capacidad operativa y de decisión.

\section{AGRADECIMIENTOS}

El grupo investigador agradece su participación a los profesionales con responsabilidades en la formación de especialistas sanitarios que participaron en los grupos de este trabajo.

Adela Alonso Abolafia, Servicio de Psicología Clínica,Hospital "Ramón y Cajal". Rocío Alvarez Nido, Medicina F amiliar y Comunitaria CS "San Blas", Área 10. Carlos Alvarez Vázquez, Servicio de Análisis Clínicos, H.U. "Doce de Octubre". J osé J avier Ariño I rujo, Servicio Anestesia y Reanimación, Hospital Clínico "San Carlos". María Angeles Balsa Bretón, Servicio de Medicina Nuclear, H.U. de Getafe. Juan I gnacio Bermejo Aycart, Servicio de Ginecología y Obstetricia, Hospital "Santa Cristina". Héctor Bueno Zamora, Servicio de Cardiología, H.G.U. "Gregorio Marañón". Luis Cabañas Navarro, Servicio de Cirugía General y Digestivo, Hospital "Ramón y Cajal". Rosa Calvo Sagarday, Hospital "La Paz". Margarita Cebrero García, Servicio de Pediatría, Hospital "Príncipe de Asturias". María Angel es Cuadrado Cenzual, Servicio Bioquímica clínica, Hospital Clínico "San Carlos". Carlos De Gregorio González, Servicio de Psiquiatría, H. Psiquiátrico de Madrid. Migual Angel Delgado Millán, Servicio de Cirugía general y digestivo, H.U. de Getafe. J osé Santiago Filgueira Rubio, Servicio de Medicina Interna, H.G.U. "Gregorio Marañón". Francisco Javier García Borda, Servicio de Cirugía General y Digestivo, H.U. "Doce de Octubre". Emilio J osé Lagarón Comba, 
Servicio de Cirugía Plástica, H.U. "Doce de Octubre". Alfredo Llovet Verdugo, Servicio de Cardiología, H.U. "Doce de Octubre". J orge Manzarbeitia Arambarri, Servicio de Geriatría, H.U. de Getafe. Alberto Marcos Dolado, Servicio de Neurología, Hospital Clínico "San Carlos". María Dolores Marín Ferrer, Servicio de Medicina Nuclear, Hospital "La Paz". J ulio Morán Morán, Servicio Traumatología y Cirugía Ortopédica, Hospital Severo Ochoa. Teresa Muñoz Yagüe, Servicio de Medicina Digestivo, H.U. "Doce de Octubre". Eva María N egroVega, Servicio de Farmacia Hospitalaria, H.U. de Getafe. Pedro Nogales Aguado, Medicina Familiar y Comunitaria CS Espronceda, Área 7. Blanca Pinilla Llorente, Servicio de Medicina Interna, H.G.U. "Gregorio Marañón". Paloma Quintana Aparicio, Servicio de Neurofisiología Clínica, Hospital "Ramón y Cajal". María Nieves Reyes Fernández, Medicina Familiar y Comunitaria Área 2. Juan Luis Rigual Pueyo, Medicina Familiar y Comunitaria CS “Las Calesas” Área 11. Asunción Rosado López, Medicina Familiar y Comunitaria CS "San Andrés" Área 11. José Luis Ruibal Francisco, Servicio de Pediatría, Hospital Clínico "San Carlos". Julián RuizMoyano Balsameda, Servicio Anestesia y Reanimación, Hospital "Ramón y Cajal". J uan Antonio Salcedo Mata, Medicina Familiar y Comunitaria EAP Vicente Soldevilla, Área 1. Raúl Sánchez González, Medicina Familiar y Comunitaria CS "San Blas" Área 10. J esús Sanz Sanz, Servicio de Medicina Interna, Hospital de la Princesa. J avier Segovia Cubero, Servicio de Cardiología, Clínica Puerta de Hierro. María Teresa Vidal Astiz, Servicio de Geriatría, H.G.U. "Gregorio Marañón". Miguel Yebra Bango, Servicio de Medicina Interna, Clínica Puerta de Hierro. Francisco J avier Zufia García, Medicina Familiar y Comunitaria CS “EI Espinillo” Área 11.

\section{BIBLIOGRAFÍA}

1. Orden Ministerial de 22 de junio de 1995 por la que se regulan las comisiones de docencia y los sistemas de evaluación de la formación de médicos y farmacéuticos especialistas. BOE 1995; 155:19793-119799 (viernes 30 de junio de 1995).
2. Boletín Oficial del Estado. Real Decreto $127 / 84$ por el que se regula la obtención de títulos de especialidades. BOE no 26, 31/1/1984.

3. The Executive Council, The World Federation for Medical Education. International standards in medical education: assessment and accreditation of medical schools'-educational programmes. A WFME position paper. Medical Education, 1998; 32:549-558.

4. De Grave, WS; Dolmans DHJ M; Van der Vleuten, CPM. Tutor intervention profile: reliability and validity. Medical Education, 1988; 32:262-268.

5. Elliot, RL; J uthani, NV; Rubin, EH; Greenfeld, d; Skelton, WD; Yudkowsky, R. Quality in residency trai ning: toward a broader, multidimensional defi niti on. Acad. Med. 1996; 71(3):243-247.

6. Borman, E; Borman E; O'Grady, P; O'Grady P. Postgraduate training. Policy paper prepared by the Subcommittee on Postgraduate trai ning: Permanent Working Group of E uropean J unior Hospital Doctors. Med E duc 1997 J an; 31(1):3-8.

7. Fernández Si erra J . Utili dad de la evaluadión naturalista para Ia formadión MIR. En: Santos Guerra MA, Prados Torres J D, Fernández Sierra J, Martín Ruiz MV, Angulo Rasco F. Evaluación Externa de la Formación de médi cos residentes: el arte de mejor ar a través del conœimiento. Barcelona: Sociedad Español a de Medicina Familiar y Comunitaria; 1996.

8. Vicent García, MD. La Formación de Espedialistas en las Instituciones Sanitarias. T ribuna Docente. 2001; 6 (2): 74 - 79.7.

9. Kitzinger J. Introduding focus groups. Brit.Med.J., 1995; 311:299-302

10. Beltran M. Cinco vías de acceso a la realidad social. En: García Ferrando M. I bañez J. Alvira F. editores. El análisis de la realidad social. Métodos y técnicas de investigación social. Madrid: Alianza,1989: 17-47.

11. Giacomini, MK; Cook, DJ . Users' guides to the medical literature. Qualitative research in Health care. Are the results of the study valid?. J AMA 2000; 284(3):357-362.

12. Giacomini, MK; Cook, DJ. Users' guides to the medical literature. Qualitative research in Health care. What are the results and how do they help me for my patients?. J AMA 2000; 284(4):478-482.

13. Sofaer, S. Qualitative methods. What are they and why we use them?. J . Health Serv. Res. 1999; 34:1101-1118.

14. Valles MS. Técnicas cualitativas de investigación social. Reflexión metodológica y práctica profesional. Madrid: Síntesis; 1997.

15. Patton, MQ. Enhancing the quality and credibility of qualitative analysis. J. Health Serv. Res. 1999; 34:110-1118. 